\title{
Technique for Digital Block Prior to Hallux Procedures
}

\section{Faith Schick, DPM, Mitesh K Patel*, MD, Homyar Karnjia, DPM, and Nicholas Taweel, DPM}

Department of Orthopaedics, Rothman Orthopaedic Institute, Sports Medicine,

Thomas Jefferson University, USA

*Corresponding Author: Mitesh K Patel, Department of Orthopaedics, Rothman Orthopaedic Institute, Sports Medicine, Thomas Jefferson University, USA.
Received: February 24, 2021

Published: March 26, 2021

(C) All rights are reserved by Mitesh K Patel., et al.

\begin{abstract}
Background: Obtaining digital anesthesia is commonly performed to facilitate procedures on the hallux. Typically, when performing a blockade of the hallux a technique utilizing multiple injection points is employed.

Methods: This technique report describes a simple approach to achieving anesthesia by means of a single subcutaneous injection beginning at the hallux interphalangeal joint. Anesthesia is obtained utilizing approximately $3 c c$ lidocaine $1 \%$. A 27 -gauge needle is used for administering the injection.

Conclusion: This technique offers a useful tool for physicians to perform a fast-acting injection during times when a patient is under stress or pain.
\end{abstract}

Keywords: Digital Block; Hallux Procedures; Anesthesia

\section{Introduction}

Local anesthesia is a technique used to induce the absence of sensation in a specific part of the body allowing patients to undergo surgical procedures with reduced pain and distress. Obtaining anesthesia by means of digital blockade is a commonly performed office procedure.

There is a multitude of literature that discusses techniques for treating ingrown nails [1-16]. The technique for achieving anesthesia necessary for these procedures, however, rarely goes into detail.

The most commonly recognized process for obtaining anesthesia of the hallux is known as an "H-Block". This technique includes a circumferential blockade of nerves surrounding the dorsal and plantar aspect of the hallux, just distal to the first metatarsopha- langeal joint. The nerves anesthetized include: Dorsally, a branch of the medial dorsal cutaneous nerve which divides into two dorsal digital branches, one that supplies the medial side of the hallux. Plantarly, the proper digital nerve of the great toe from the medial plantar nerve supplies the skin on the medial side of the great toe. Along the first interspace, the medial terminal branch of the deep peroneal nerve divides into two dorsal digital nerves which supply the adjacent sides of the hallux and second toe [17].

Typically, when performing a blockade of the hallux multiple injections may be employed to achieve the desired anesthesia necessary to perform a partial or total nail avulsion. This may lead to loss of proprioception when the patients ambulate following the procedure [17].

Alternatively, a partial blockade of the hallux may be utilized when the patient presents with a more focal problem requiring

Citation: Mitesh K Patel., et al. “Technique for Digital Block Prior to Hallux Procedures". Acta Scientific Orthopaedics 4.4 (2021): $64-67$. 
treatment isolated to either the medial or lateral margin of the hallux.

Multiple factors will affect the level of pain associated with achieving digital blockade: the needle diameter, the angle of penetration, the number of needle sticks, the temperature and type of solution including $\mathrm{pH}$, and the rate of injection [18].

This technique describes a proper digital block performed beginning just distal to the level of the interphalangeal joint. The technique represents an effective means of obtaining digital blockade while reducing the quantity of anesthetic given and increasing time to onset of anesthesia. The ability to perform a single, fast-acting injection serves as a useful tool during times when a patient is under stress or pain. The purpose of this paper is to add to the limited literature available discussing injection technique in the foot and ankle.

\section{Procedure technique}

This technique describes a step-by-step process for achieving local anesthesia to a hallux single nail margin via one injection. Anesthesia is obtained utilizing approximately $3 \mathrm{cc}$ lidocaine $1 \%$. A 27-gauge needle is used for administering the injection.

The injection is given beginning just proximal to the hallux interphalangeal joint.

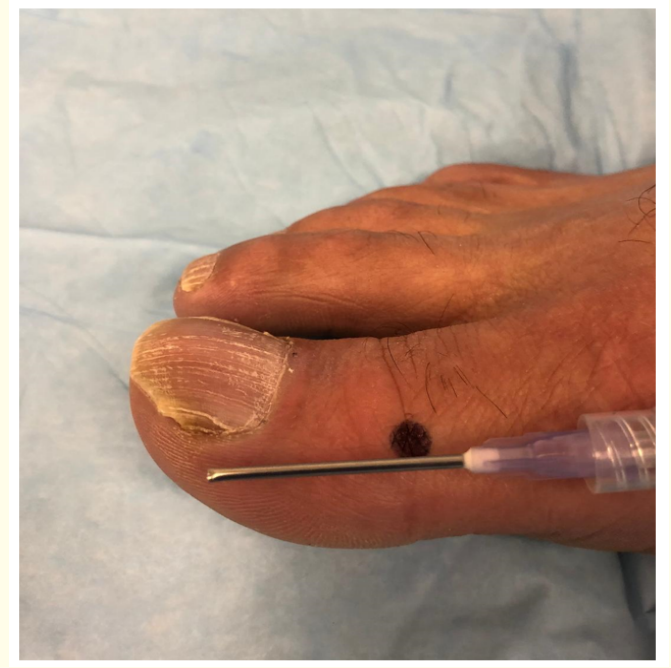

Figure 1: Injection is begun by raising a small wheel and proceeding from a dorsal to plantar direction at the level of the interphalangeal joint.
A wheal is raised initially. Then, a direct dorsal to plantar approach is used to dispense approximately 1cc of lidocaine. The needle is then withdrawn slightly and angulated in a proximal to distal fashion.

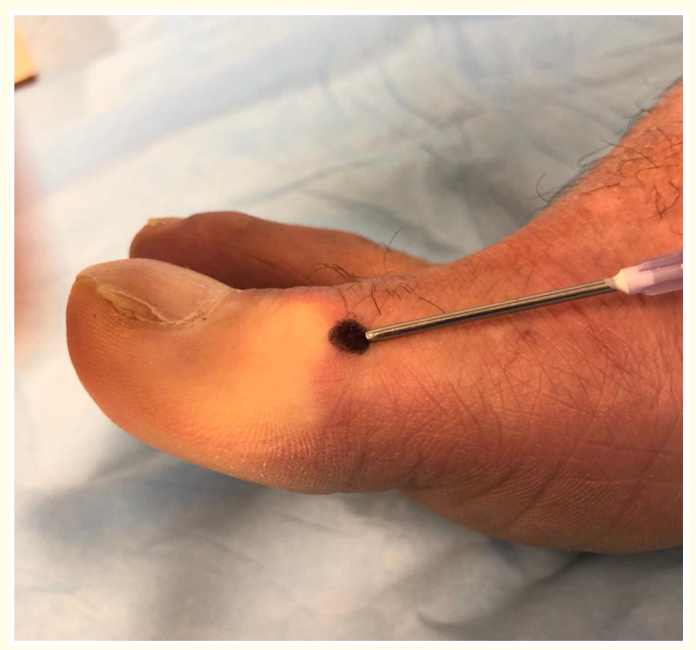

Figure 2: The needle is then angulated and the injection is continued subcutaneously from a proximal to distal fashion.

The remainder to the solution is dispensed subcutaneously until blanching of the surrounding nail fold tissue is achieved.

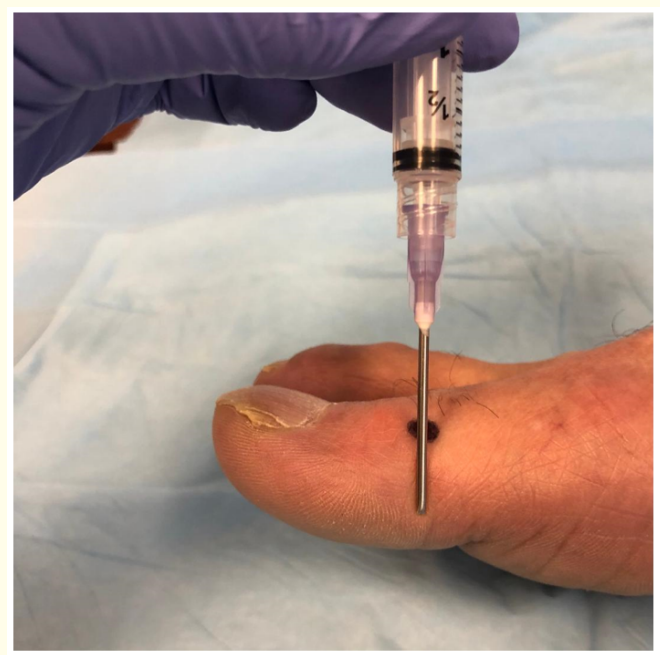

Figure 3: Lidocaine is infiltrated until blanching of the tissue occurs along the affected nail margin. 


\section{Discussion}

It was found that utilizing this technique allowed for minimal complaints discomfort during injection. Anesthesia was achieved quickly allowing the physician to begin the partial nail avulsion immediately following the injection. Additionally, in all cases anesthesia was achieved utilizing $3 \mathrm{cc}$ or less of $1 \%$ lidocaine.

Serour., et al. studied the efficacy of Eutectic mixture of local anesthetic (EMLA) cream (lidocaine 2.5\% and prilocaine 2.5\%) application prior to digital ring blocks for surgery for ingrown hallux nails. The prospective, double-blinded, placebo-controlled, randomized clinical trial looked at a total 81 patients. They found there to be no clinical benefit in using EMLA cream during digital nerve block $(\mathrm{P}<0.005)[19]$.

In 2010, Whitely., et al. completed a randomized controlled trial comparing two techniques for partial digital local anesthetic blocks. In this study, they were able to demonstrate a highly statistically significant preference amongst patients using a two stage injection method. The technique involved an initial injection advanced from the dorsal to plantar aspect of the toe and then withdrawn releasing a quantity of approximately $0.2 \mathrm{ml}$ of anesthetic. After a two-minute interval, the remaining $0.8 \mathrm{ml}$ of anesthetic was administered to the same site to the dorsal and plantar nerves. The two stage technique was associated with less intense pain of a shorter duration than the traditional one stage injection method [20].

More studies have been performed looking at injection technique for finger blockade. Williams., et al. in 2006 described a technique for single injection in the midline of the proximal phalanx using lidocaine and epinephrine. They introduced the term SIMPLE (single subcutaneous injection in the midline of the proximal phalanx with lidocaine and epinephrine). This study demonstrated a preference amongst patients for the SIMPLE block over the traditional two dorsal web space injection technique [21].

Additional orthopedic literature has looked at the rate of injection and found conflicting evidence as to whether or not there is a decrease in pain with a slower rate of injection $[18,22,23]$. A Cochrane review recommended adjusting the $\mathrm{pH}$ of lidocaine with epinephrine to 7.4 [24]. In a study by Hogan., et al. systematic review and meta-analysis was performed looking at the effect of warming local anesthetics and found injections to be less painful [25].
This paper was intended to describe a variation on the technique normally utilized to achieve a partial hallux blockade. Additional consideration may be given when utilizing our single injection technique to employ adjustments for temperature, $\mathrm{pH}$ and rate of injection. Future analysis of this technique may also involve a controlled trial in which the technique is compared to a standard partial hallux block while measuring pain on a visual analog scale.

This technique has proven to be a successful means of achieving anesthesia in the hallux for performing partial nail avulsions. It allows for quick and less painful onset of anesthesia for patients. This is of particular interest when treating pediatric patients in which it may be difficult to obtain a multiple point injection due to movement and discomfort. A single injection technique is also favorable when patients are already experiencing stress and pain from ingrown nails.

\section{Conclusion}

This technique offers a useful tool for physicians to perform a fast-acting injection during times when a patient is under stress or pain.

\section{Bibliography}

1. Khan IA., et al. "Treatment of ingrown toe nail-comparison of phenolization after partial nail avulsion and partial nail avulsion alone". Journal of Ayub Medical College Abbottabad 26.4 (2014): 522-525.

2. Eekhof JA., et al. "Interventions for ingrowing nails". The Cochrane Database of Systematic Reviews 4 (2012): CD001541.

3. Antrum RM. "Radical excision of the nailfold for ingrowing toenail". The Journal of Bone and Joint Surgery British 66 (1984): 63.

4. Aksakal AB., et al. "Decompression for the management of onychocryptosis". Journal of Dermatological Treatment 15 (2004): 108.

5. Alptekin H., et al. "Simple new operative technique to treat ingrown toenails without recurrence". The European Journal of Dermatology 21 (2011): 281.

6. Bose B. "A technique for excision of nail fold for ingrowing toenail". Surgery, Gynecology and Obstetrics 132 (1971): 511. 
7. Noel B. "Surgical treatment of ingrown toenail without matricectomy". Dermatologic Surgery 34 (2008): 79.

8. Persichetti P., et al. "Wedge excision of the nail fold in the treatment of ingrown toenail". Annals of Plastic Surgery 52 (2004): 617.

9. Haricharan R., et al. "Nail-fold excision for the treatment of ingrown toenail in children". The Journal of Pediatrics 162 (2013): 398.

10. El-Shaer WM. "Lateral fold rotational flap technique for treatment of ingrown nail”. Plastic and Reconstructive Surgery 120 (2007): 2131.

11. Karaca N and Dereli T. "Treatment of ingrown toenail with proximolateral matrix partial excision and matrix phenolization". Annals of Family Medicine 10 (2012): 556.

12. Winograd AM. "Modification in the technique of operation for ingrown toenail". The Journal of the American Medical Association 92 (1929): 229.

13. Suppan RJ and Ritchlin JD. "A non-disabilitating surgical procedure for ingrown toenail". Journal of the American Podiatric Medical Association 52 (1962): 900-902.

14. Yale JF. "Phenol-alcohol technique for correction of infected ingrown toe-nail". Journal of the American Podiatric Medical Association 64 (1974): 46-53.

15. Zuber TJ. "Ingrown Toenail Removal”. American Family Physician 65 (2002): 2547-2550.

16. Erdogan FG. "A simple, pain-free treatment for ingrown toenails complicated with granulation tissue". Dermatologic Surgery 32 (2006): 1388-1390.

17. Khan K. "Local Anesthesia Techniques - These Injections are commonly used in podiatric surgery". Podiatry Management (2017): 152-159.

18. Hamelin ND., et al. "Decreasing the pain of finger block injection: level II evidence”. Hand 8.1 (2013): 67-70.

19. Serour F., et al. "EMLA cream prior to digital nerve block for ingrown nail surgery does not reduce pain at injection of anesthetic solution". Acta Anaesthesiologica Scandinavica 46.2 (2002): 203-206.
20. Whitley B and Rees S. "A Randomized controlled trial to compare two techniques for partial digital local anesthetic blocks". The Journal of Foot and Ankle Surgery 49.2 (2010): 143-146.

21. Williams JG and Lalonde DH. "Randomized comparison of single-injection volar subcutaneous block and the two-injection dorsal block for digital anesthesia". Plastic and Reconstructive Surgery 118.5 (2006): 1195-1200.

22. Krause RS., et al. "The effect of injection speed on the pain of lidocaine infiltration". Academic Emergency Medicine 4.11 (1997): 1032-1035.

23. Whitworth JM., et al. "Influence of injection speed on the effectiveness of incisive/mental nerve block: a randomized, controlled, double blinded study in adult volunteers". Journal of Endodontics 33.10 (2007) 1149-1154.

24. Cepeda MS., et al. "Adjusting the pH of lidocaine for reducing pain on injection". Cochrane Database of Systemic Reviews 8.12 (2010): CD006581.

25. Hogan ME., et al. "Systematic review and meta-analysis of the effect of warming local anesthetics on injection pain". Annals of Emergency Medicine 8.1 (2011): 86-98.

\section{Assets from publication with us}

- Prompt Acknowledgement after receiving the article

- Thorough Double blinded peer review

- Rapid Publication

- Issue of Publication Certificate

- High visibility of your Published work

Website: www.actascientific.com/

Submit Article: www.actascientific.com/submission.php Email us: editor@actascientific.com

Contact us: +919182824667

Citation: Mitesh K Patel., et al. “Technique for Digital Block Prior to Hallux Procedures". Acta Scientific Orthopaedics 4.4 (2021): 64-67. 